\title{
An overview of the holographic display related tasks within the European 3DTV project
}

Levent Onural

Haldun M. Ozaktas

Elena Stoykova Atanas Gotchev John Watson 


\title{
An Overview of the Holographic Display Related Tasks within the European 3DTV Project
}

\author{
Levent Onural ${ }^{1}$, Haldun M. Ozaktas ${ }^{1}$, Elena Stoykova ${ }^{2}$, Atanas Gotchev $^{3}$, John Watson ${ }^{4}$, \\ ${ }^{1}$ Bilkent University, Department of Electrical and Electronics Engineering \\ TR-06800 Bilkent, Ankara, Turkey \\ ${ }^{2}$ CLOSPI-BAS \\ Sofia-1113, Acad. G. Bontchev St. - 101, P.O. Box 95, Sofia, Bulgaria \\ 3 Tampere University of Technology, Institute of Signal Processing \\ FIN-33101 Tampere, Finland \\ ${ }^{4}$ Department of Engineering, Universty of Aberdeen, King's College \\ AB24 3FX, Scotland, UK
}

\begin{abstract}
A European consortium has been working since September 2004 on all video-based technical aspects of threedimensional television. The group has structured its technical activities under five technical committees focusing on capturing 3D live scenes, converting the captured scenes to an abstract 3D representations, transmitting the $3 \mathrm{D}$ visual information, displaying the $3 \mathrm{D}$ video, and processing of signals for the conversion of the abstract 3D video to signals needed to drive the display. The display of $3 \mathrm{D}$ video signals by holographic means is highly desirable. Synthesis of high-resolution computer generated holograms with high spatial frequency content, using fast algorithms, is crucial. Fresnel approximation with its fast implementations, fast superposition of zonelens terms, look-up tables using pre-computed holoprimitives are reported in the literature. Phase-retrieval methods are also under investigation. Successful solutions to this problem will benefit from proper utilization and adaptation of signal processing tools like waveletes, fresnelets, chirplets, and atomic decompositions and various optimization algorithms like matching pursuit or simulated annealing.
\end{abstract}

Keywords: 3DTV, holographic TV, dynamic holographic displays

\section{TECHNICAL SCOPE OF THE 3DTV PROJECT}

A European consortium has been working since September 2004 on all video-based technical aspects of threedimensional television (3DTV) [1]. The group has structured its technical activities under five technical committees. These technical committees are focusing on capturing 3D live scenes, converting the captured scenes to abstract 3D representations, transmitting the 3D visual information, displaying the 3D video, and processing of signals for the conversion of the abstract $3 \mathrm{D}$ video to signals needed to drive the display. The technical scope is kept rather wide: the researchers are investigating many different competing technologies for each one of the technical components of the overall 3DTV system in a comparative approach. Fig. 1 shows the main functional blocks.

Multi-camera systems are quite popular among the alternative approaches to acquire 3D moving scene information. Typical multi-camera systems have 2-20 cameras providing synchronized video streams. Calibration is an issue. Typically, the images of a point in the $3 \mathrm{D}$ scene at an instant is located in more than one view captured by the cameras, and the true 3D physical coordinates are then computed from this information. Automated matching of feature points is a research topic. Holographic recording of 3D scene information using digital cameras is also under investigation as a candidate technology.

Once the 3D geometry is captured, together with its color texture, the captured scene can be represented using various computer graphics techniques; for example, mesh polygons may be used.

This work is supported by EC within FP6 under Grant 511568 with acronym 3DTV.

Photon Management II, edited by John T. Sheridan, Frank Wyrowski, Proc. of SPIE Vol. 6187,

61870T, (2006) · 0277-786X/06/\$15 - doi: 10.1117/12.668540 


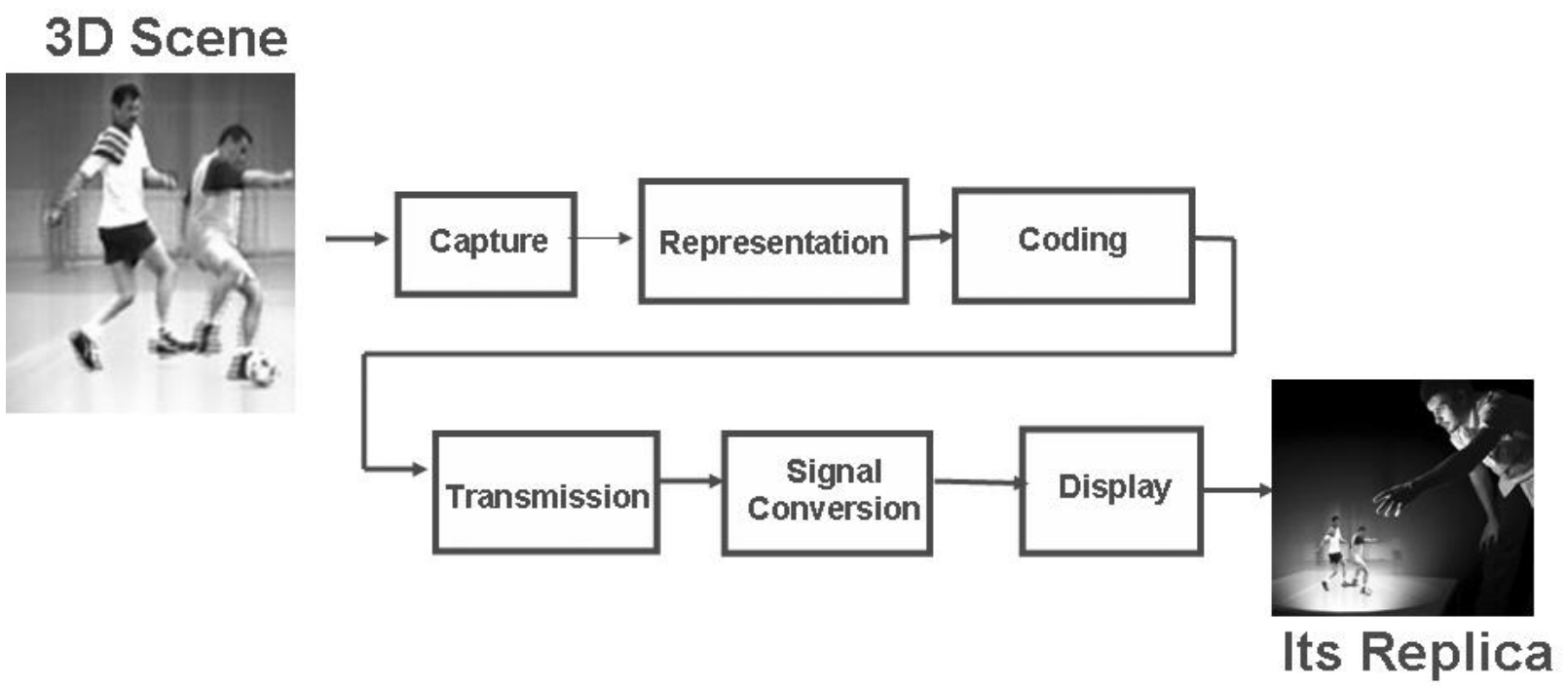

Figure 1. The main technical components of 3DTV

An important step is the compression and coding of the captured 3D motion pictures. Compression of 2D video has been well studied during the past three decades. However, extensions to stereo-video, or multi-view video, are rather new. ISO MPEG activities for standardization of multi-video data is still ongoing. Direct compression of 3D video using mesh-compression techniques is also under investigation.

Real-time delivery of captured, compressed data is another task within the 3DTV project. Transport over Internet Protocol (IP) architectures based on packet network technology is being considered.

Naturally, the user interaction with the 3DTV will be via the 3DTV display. There are many technologies at different levels of maturity. Stereoscopy is an almost 100-years old technology for the display of 3D motion pictures. Autostereoscopic techniques which do not require any eye-wear to observe 3D are also common. Headtracking stereo displays provide a more pleasant viewing experience by tracking the location of the observer, and then by displaying the proper pairs of the stereo for the captured location. Holographic displays are far from being mature at this time. However, interest in holographic displays is increasing together with recent developments in the underlying electronics and optical technologies necessary for the satisfactory operation of such displays.

A difficult problem is the conversion of captured 3D scene geometry and texture information to optical signals in space such that when observers receive those optical signals, they perceive the underlying 3D scene. Computational techniques which will convert the given 3D scene information to 3D propagating light waves in 3D space are under investigation. Furthermore, once the desired 3D propagating light distribution is found, another difficult problem is the computation of the driving signals of a physical device which would have the capability to create such 3D optical fields. Naturally, the second problem depends on the physical structure and limitations of the candidate device.

In this paper, we will focus on certain aspects of holographic displays, and the associated signal processing techniques needed for the solution of the two already mentioned fundamental problems: a) Given a 3D object, with its structure and texture, compute the 3D complex light field which would emanate from such an object, b) Given a 3D complex light field, and a physical device which is capable of generating such 3D fields with a given degrees of freedom, find the (electronic) signals to drive the device. Naturally, the ultimate problem is the concatenation of the two problems above: given the 3D object and the field-generating source device with its input/output characteristics and limitations, how do we drive the device to obtain the desirable effect. Side problems will follow: under the constraints imposed on the physical device, what will be the quality of the viewed best $3 \mathrm{D}$ reconstruction of the object. 
Candidate devices for the display are various transmittive and reflective spatial light modulators (SLM), including deflectable mirror array devices (DMAD). Commercially available versions are of utmost interest $[2,3]$.

\section{ISSUES IN HOLOGRAPHIC 3DTV DISPLAYS}

The display of captured and transmitted 3DTV video signals by holographic means is highly desirable since holography is capable of creating realistic images with all the depth cues. A full parallax, large area, interactive, moving, colour holographic display, which is thought by many to be the ideal goal, requires incremental and parallel development of many essential areas of technology before it can be brought to fruition. For example, a large display of say $100 \mathrm{~mm}$ diagonal will need dramatic improvements in VLSI techniques to enable an SLM to be manufactured with sufficient pixel resolution. If the oft-quoted "Moore's Law" continues to apply then it could still be more than 8 years before a display of less than micron pixel size is achieved. An array of SLMs requires advances in interconnection technology and software required to drive them. Colour displays require development of compact, safe lasers or LEDs with sufficient coherence and power. Synthesis of high-resolution computer generated holograms (CGHs) with high-spatial frequency content for 3D object presentation is most crucial for creation of true 3D perception of real-world scenes with randomly distributed diffuse objects. The task of CGH synthesis is particularly challenging in the case of dynamic displays which require fast approaches for simulation of the underlying physical phenomena to enable refresh rates of the consecutive display frames suitable for real-time representation. Given the present state of modern computer power and capacity, such an approach should rely on some approximation technique and corresponding fast algorithms for its implementation.

Reconstruction of 3D images from a high-spatial-frequency content CGH is usually based on the FresnelKirchoff integral whose computation is a time-consuming procedure. This fact has motivated a large amount of research in order to facilitate calculation of the complex amplitude in the hologram plane (the SLM plane). In the mainstream of investigation efforts, a separate branch is formed by Fourier or Fresnel transform based approaches allowing for numerical implementation by the FFT algorithm. They rely on the object presentation as a collection of parallel or tilted planar projections or slices. The "ping-pong" algorithm for solid opaque objects that is implemented by a cascade of Fourier transforms $[4,5]$ in planes perpendicular to the optical axis has been followed by calculation of the complex amplitude through subdividing the $3 \mathrm{D}$ object in tilted planar segments with a given amplitude distribution and superposition of the corresponding diffractive structures in the plane of the hologram [6,7], reconstruction of the 3D image on an arbitrary curved surface from a Fourier hologram [8], and viewing of the 3D object from a changing angle [9]. To avoid the usage of light interference as main limitation of holographic information acquisition, a method was developed of recording a CGH of a real-world 3D object under conditions of incoherent white illumination with further true wave front reconstruction under coherent illumination [10] achieved by computing of an equivalent 2D presentation of the complex amplitude of coherent light diffracted from the object using 1D Fourier transforms and a particular optical system with two cylindrical lenses. This idea is further developed in [11] where synthesis of lensless Fresnel holograms is proposed by calculation of 3D Fourier spectra based on tomographic acquisition of different perspectives of the 3D object recorded by use of an incoherent light source and extraction of spectral components for generation of a limited viewing area hologram. The method is further extended to reconstruct full color images from three CGHs synthesized from a 3D Fourier spectrum obtained from several projection images recorded under incoherent illumination [12]. The main drawback of the methods proposed in [11-13] is the time-consuming process of information capture in the case of real objects by 2D scanning that requires precise control of the CCD camera and gives redundant data. The solution of the problem is found in [14] by synthesis of the CGH in the 3D Fourier space using only (1D) azimuth scanning. A Fourier transform based algorithm is developed in [15] for fast calculation of diffractive structures that permit image reconstruction on cylindrically and spherically curved surfaces. At this stage of research, the Fourier transform based algorithms are mostly used for generation of static holograms for 3D object presentation; however, they deserve a special attention as a basis for development of reliable phase-retrieval techniques.

More preferable for realization of an interactive holographic display is another popular approach of CGH calculation through a superposition of analytic distributions (e.g., Fresnel zone plates). This is equivalent to Kirchoff diffraction integral approximation by decomposing the object into a certain number of discrete independent point sources, line segments or higher-order image elements. The modeled underlying physical phenomenon is 
the interference between the light waves coming from the analytically defined "holoprimitives" constructing the object and the reference wave to form the resulting complex amplitude distribution on the hologram plane [16]. This interference-based approach puts calculation of the CGHs on a hardware-based ground by imitating the interference of a pre-computed set of complex textures [17-19] that describe the object or by real-valued linear summation of pre-computed elemental fringes corresponding to a given holoprimitive stored in a "look-up" table $[20,21]$. Representation of object/image elements at different locations by scaling and translation of similar elemental diffractive structures in the plane of the hologram permits fast updating of the CGH by the so called incremental computing approach, which incorporates not only depth information and continuous parallax, but also permits one to apply standard rendering techniques such as hidden-surface elimination or surface shading [22]. Real-time CGH computation has been proposed by introducing holographic encoding techniques such as "hogel-vector" and "fringelet" encoding on the basis of spatial and spectral subsampling of Wigner distribution without simulation of the interference in which the complex amplitude in the SLM plane is obtained by linear summation of pre-computed basis fringes with specific diffractive functions that have been beforehand calculated and stored in a look-up table [23]. The high ratio of bandwidth compression achieved by this encoding technique to increase the computational speed inevitably expands the image point spread function thus causing speckle-like images. For this reason this approach is less preferred in existing prototypes of dynamic holographic displays.

Due to the high-quality of the images yielded by the interference-based calculation and the possibility for its hardware implementation, this technique has been widespread for CGH computation in recently reported dynamic holographic 3D display systems [22,24-28]. For acceleration of the CGH computation, the full parallax or the dependence of amplitudes of the elemental waves emitted from the different object points on the distance between them and sampling points on the CGH is often omitted. Nevertheless, the computational burden imposed by the large number of object points and the requirement for high spatial and angular resolution of the obtained images remains the main obstacle in the realization of the dynamic holographic display that should be eliminated by further improvement or more effective solutions.

A solution worthy to be considered is to seek a possibility to combine capture of information with direct creation of the required diffractive structure in the hologram or the SLM plane. For 3D scene capture one may use phase retrieval techniques developed for optical profilometry which yield information about the object without sophisticated iterative algorithms. One of the simplest phase-retrieval approaches is pattern projection with incorporation of phase-shifting technique. This approach is especially suitable for outdoor recording of real scenes due to the possibility to use incoherent white light illumination. It is also characterized with less vulnerability to external vibration than the traditional interferometric or holographic approaches. However, although permitting real-time capture of a real 3D scene under incoherent illumination, the recorded patterns can not be fed directly to the SLM, even in the case of interferometrically projected fringes. The derived 3D coordinates could be input data for the traditional interference-based calculation with all its shortcomings. The idea to use a traditional holographic approach with an object and a reference wave for remote capture of information about the 3D objects that is followed by direct feeding of the recorded interference pattern to the SLM in the holographic display is described in [29]. The main drawback is the resolution of the existing dynamic SLMs as LCDs or DMDs which is not enough for reconstruction of the 3D images with high quality. Only AOM SLMs could provide such a resolution in the Bragg diffraction mode but they are one-dimensional structures which entails omitting of the vertical parallax.

From a signal processing point of view the task of direct determination of the complex amplitude of the object wavefront in the plane of the SLM by employing a set of intensity measurements is a phase-retrieval problem. In general, the problem of phase-retrieval from intensity measurements of optical signals has received a large amount of attention [30,31]. Among the more studied variations is phase-retrieval from space- and frequency-domain intensity information, recently generalized to arbitrary fractional Fourier transform intensities and further generalized to multiple fractional Fourier transform intensities. This approach requires measurement of intensity in multiple planes (at least two). Increasing the number of planes in which we are measuring the signal contributes to robustness. The single measurement problem in which we have only the Fourier transform intensity, with additional constraints such as finite extent, non-negativity etc., also deserves attention. Less studied but of special interest in the present context are the methods which employ multiple intensity measurements obtained by varying the input in some manner. This can be either deliberate or exploit naturally occurring variations. 
Actually, most methods of phase-retrieval are based on obtaining the intensity of two different versions of the signal which constitute independent equations which can then be solved iteratively or by other means to obtain the phase. The problem is intrinsically non-convex so that iterative algorithms require some care.

Generally speaking, most phase-retrieval approaches involve measurement of intensity distributions, from which the amplitude and phase of the wavefront are reconstructed, a process which parallels the holographic process. This correspondence does not seem to have received very widespread attention. A speculation can be made that, if an algorithm or method is known that can retrieve the phase from a certain set of derived signals and auxiliary information, then that set of derived signals and auxiliary information can be taken as the "hologram" of the original signal. The most common "derived signal" is the intensity of some transformed or otherwise modified version of the original signal. Therefore, the intensity recording of a signal and its Fourier transform or any of its fractional Fourier transforms, taken together, constitute a hologram of the signal. Other sets of information from which the phase can be retrieved can be obtained by modifying, modulating, masking, etc. the original signal before recording intensities. The intensity produced by these modified inputs can then be used to retrieve the phase and thus constitute a hologram of the signal. Notice that many of these methods rely on digital retrieval, not optical retrieval. Based on the above considerations, a concrete research direction may be put forth as search of such sets of derived signals that are candidates for being "holograms" of the original signal. In fact, it is possible to invent many ways of modifying a signal to produce more than one independent intensity measurement. Such approaches may involve shifting, tilting, modulating, masking, and parametrically transforming the original signal.

\section{SIGNAL PROCESSING TOOLS FOR HOLOGRAPHIC 3DTV}

Techniques to generate holograms for 3DTV display purposes, as outlined in the previous section, heavily rely on signal processing tools. That includes not only the algorithms for the solution of the problems presented above, but also fundamental issues associated with discrete representations of propagating waves. For example, consideration of sampling of such signals yields rather unexpected theoretical results for the sampling rate requirement for complete recoverability of the underlying continuous signals $[32,33]$. The association between the fractional Fourier transform and Fresnel diffraction paves the way for importing related signal processing results directly to the solutions of the above mentioned fundamental problems [34]. Yet another strong link between signal processing and optical wave propagation is the direct import of some modern signal processing techniques like, wavelets, atomic decompositions, fresnelets, beamlets, etc. Furthermore, it is certain that useful solutions will inevitably require the application of basic results of information theory to the problems above, including the presence of noise and quantization.

Naturally, in addition to the basic theoretical issues, associated fast algorithms for feasible operation are of interest. Furthermore, engineering approximations are needed, and therefore, both such approximations, and their assessment are among the open problems. However, there are currently existing fast algorithms which can solve some rather simpler basic problems somewhat related to the stated problems [35]. Extensions to the color case remain challenging.

An outline of certain signal processing tools associated with holographic 3DTV display related problems is given below.

\section{Efficient signal representations and optimization algorithms}

Abstract structures are best described in terms of appropriate coordinate systems; i.e., the object of interest is represented by its (discrete) coordinates in some well-defined function space. Modern sampling and approximation theories have addressed the problem of defining such spaces, such as translation-invariant spaces, multiresolution spaces, etc. and equipped them with the corresponding bases or frames [36,37]. Then the signal representation problem is formulated as finding the best signal expansion on an alternative coordinate system. Such "projection" approaches have been shown to be useful in many signal processing areas such as approximation, feature extraction, compression, noise and blur removal, and regression. A change of the basis system allows to capture the signal characteristics by a fewer number of significant coordinates, and consequently to make the processing simpler and computationally less demanding. 
A number of basis systems, denoted as "dictionaries of atoms" designed and studied with different purposes can be found in the literature. Among them are wavelet and cosine packets, Gabor functions, curvelets, ridgelets, beamlets, chirplets, fresnelets, etc. Also, optimization strategies for finding the best representation, such as bestbasis search, matching pursuit, and basis pursuit, have been designed within the same abstract framework.

The reason for emphasizing the so-called "atomic decomposition approach" for the holographic display problem area is two-fold. First, some basis dictionaries such as Gabor wavelets, chirplets and fresnelets have already proved useful in processing and reconstruction of digital holographic signals [38]. Second, basis construction methodologies can be used or modified to design new basis families suitable for the problems in holography and relevant optimization techniques can be applied to solve the computationally-burdened inverse problems in hologram synthesis.

Below, we review some of the basis systems and relate them to the problems we are dealing with.

\section{Wavelets}

The theory of wavelets is a branch of harmonic analysis and an effective tool for solving various signal processing problems. Wavelets are functions that are well localized in the time (or space) domain or in the frequency domain and offer an optimal tradeoff between time (space) and frequency (scale) resolution. Therefore they are convenient to represent both stationary and transient signals.

Wavelets are basis functions that separate the $L^{2}(\mathbf{R})$ space of square integrable functions to a collection of nested subspaces. Starting from $L^{2}(\mathbf{R})$, the separation is made stepwise by separating each subspace to a direct sum of subspaces: one representing coarser approximations, and the other being the difference between the coarse approximation subspace and the parent space. This quite formal construction has strong relation with the theory of digital filters and filter banks. Filter banks are involved in the design of wavelet families [39] and the projection of a function on the nested wavelet spaces is done through discrete algorithms utilizing filter banks [40]. The separation between subspaces is equivalent to frequency subband analysis.

Contemporary wavelet theory is about 20 years old but is already considered classic. We refer to an excellent review by Benedetto [41] where the history, major research achievements and implications of the theory of wavelets are reviewed in a fascinating way.

Being able to capture both stationary and local behaviour of the signal, wavelets have found application in many signal processing areas - compression, noise removal, deconvolution, pattern recognition and detection, prediction and regression. In [42] and [43] it is shown how wavelets can be used for analysis and reconstruction of in-line holograms. In [42] the wavelet transform is used to reconstruct successive planes of a sample volume and find the 3D location of small particles. The purpose of the work [43] is to determine the three-dimensional trajectories of glass fibers drawn out in a turbulent flame, and high-speed in-line holography is used to visualize the sample volume. With the help of wavelets a noiseless, selective, and fast reconstruction of the 3D field is performed.

Overcomplete and sparse atomic representations and expansions on dictionaries of bases

By dictionary $D$ we denote a collection of parameterized waveforms or atoms $\left\{\phi_{\gamma}\right\}_{\gamma \in \Gamma}$, with a parameter $\gamma$. A signal $\mathrm{s}$ is decomposed as:

$$
s=\sum_{i=1}^{m} \alpha_{\gamma_{i}} \phi_{\gamma_{i}}+R^{(m)},
$$

where $R^{(m)}$ is the signal residual after decomposing on $m$ dictionary elements. In the case when $R^{(m)}=0$ the signal has exact representation in the dictionary $D$.

Most dictionaries are overcomplete: they are overcomplete either by their nature, or because they merge various complete dictionaries. In the latter case, the new mega-dictionary consists of several types of waveforms (e.g., Fourier and wavelet dictionaries combined). The decomposition given by Eq. 1 is then non-unique, as some elements in the dictionary have representations in terms of other elements. Non-uniqueness gives the possibility of adaptation; i.e., of choosing among many representations one that is most suited to our purposes. Usually, the sparsest possible representation, i.e., that with fewest significant coefficients is targeted. Also, speed is an essential issue: it should be possible to obtain a representation in order $O(N)$ or $O(N \log N)$ computations. 
Collections of waveforms providing sparse representations and fast computation for various classes of signals are briefly reviewed below.

\section{Wavelet and cosine packets}

Wavelet and cosine packets are a collection of functions that are well localized in the time (space) domain or in frequency (scale) domain or in both $[40,44]$. They are convenient for description of important signal features such as edges, spikes, transients or oscillations.

The wavelet packets are constructed by dividing the frequency plane to regions. To each frequency band corresponds a frequency-indexed prototype function. Dyadic dilations and translations of the prototype functions determine all the elements in the dictionary. A complete signal decomposition is found in $O(N)$ operations with Mallat's filter bank algorithm [40].

The local cosines are constructed by dividing the spatial domain to blocks. For each block a basis of windowed smooth trigonometric functions is built. In the discrete domain there are fast implementations involving existing fast DCT algorithms.

Wavelet and local cosine packets have been applied in all areas of signal processing. Allowing fast signal adaptation they might be useful also for digital holography.

\section{Gabor wavelets}

The Gabor wavelet dictionary consists of frequency scaled and rotated Gabor functions that are able to extract directional features from multidimensional signals $[45,46]$. In [38] the authors have shown that the Gabor wavelets are optimal for processing and reconstructing holograms. In order to prove optimality, they derive an uncertainty principle for the energy spread of a function in the sense of the Fresnel transform. This principle is analogous to the Heisenberg uncertainty relation derived for the Fourier transform. The energy spread is defined as the product between the variance of a function in time (spatial) domain and its variance in Fresnel domain. Gabor-like functions turn out to have minimal energy spread for the Fresnel transform.

\section{Chirplets}

A chirplet is a compact support signal with increasing (decreasing) frequency [47], [48]. A widely-used chirplet family is constructed by modulating a Gaussian envelope with a linear chirp oscillation. Therefore chirplets are rather attractive for representation of holograms since they have minimal energy spread for the Fresnel transform in the same sense as Gabor functions.

The family of Gaussian chirplets are determined by four parameters: time width $\Delta$, time center $\tau$, frequency center $f$, and chirp rate $c$. The construction of the bases starts with computation of the time-frequency (TF) distribution of a "mother" chirplet $g(t)$ which is usually a Gabor wavelet (constant frequency). Then a chirplet dictionary can be generated through some geometrical transformations of $g(t)$ in the TF plane such that the whole TF plane is covered [48]. These transformations are time translation $g(t-\tilde{\tau})$, frequency translation $e^{j 2 \pi \tilde{f} t} g(t)$, dilation in time/frequency $g(t / \tilde{\Delta})$ and shearing $e^{j \pi \tilde{c} t^{2}} g(t)$ (increase of the chirprate by $\tilde{c}$ ).

References $[49,50]$ and [51] suggest methods that use the matching pursuit framework to find the parameters of a sparse chirplet signal representation. The method in [49] determines the parameters $\Delta, \tau, f$ and $c$ of each atom iteratively, starting from $c=0$ and range for the others computed using the zooming algorithm [52]. However, this iterative procedure is very demanding since it requires computation of tens of inner products for determining a single atom in the representation 1. A recent work of the same authors [50] suggests a method for optimal determination of the chirplet parameters that involves only six inner products. The basic idea there is to convert the optimization process to a traditional curve-fitting problem by solving a group of equations obtained on a set of different testing points. In earlier work Grobonval achieves also an $O(M N)$ algorithm for estimating the optimal parameters, using a fast algorithm to estimate the best Gabor atom $(c=0)$ and the local behaviour of $f$ around the optimal value to compute the other parameters [51]

Chirplet transforms can be useful in digital holography since it is known to be a good instantaneous frequency estimator [53]. This is important in areas where electromagnetic interference is present. Significant examples are represented by the analysis of radar signals and seismic reflections. 


\section{Fresnelets}

Fresnelets are wavelet-like bases that are constructed for the reconstruction and processing of Fresnel holograms recorded on CCD-arrays.

Since it is a lensless process, digital holography tends to spread out sharp details such as object edges over the entire imaging plane. Standard wavelets, typically designed to represent piecewise smooth signals give poor results when applied directly to the hologram. The approach in $[38,54]$ is to apply a Fresnel transform to a wavelet basis of $L^{2}(\mathbf{R})$ to simulate the propagation in the hologram formation process and thus to build an adapted fresnelet basis. As pointed in the same work the Fresnel transform (which is unitary) maps a Reisz basis into a Reisz basis with the same Reisz bounds. Moreover, the Fresnel transform preserves the embededness of the subspaces spanned by different basis functions. However, in classical wavelet theory embedded multiresolution spaces are generated through dilation of one single function. The difference here is that in the transformed domain there is one generating function for each scale.

B-spline biorthogonal wavelets are used to construct the fresnelet dictionaries. They are well localized in the sense of the uncertainty principle for the Fresnel transform and have excellent approximation characteristics. Another good feature is that they have analytic expressions in the time domain and the Fresnel transform of them and their associated wavelets can be derived explicitly [38]. The fresnelet transform allows for the reconstruction of complex scalar waves at several user-defined, wavelength-independent resolutions. Furthermore, when reconstructing holograms numerically, the subband decomposition of the fresnelet transform naturally separates the image to reconstruct from the unwanted zero-order and twin image terms.

\section{SUMMARY AND CONCLUSION}

In this paper we have provided an overview of some of the holographic display related tasks within the European 3DTV project, emphasizing some of the key problems arising in holographic 3DTV displays and important tools set forth for their solution. We provided a general overview of the technical scope of the whole project to put the concerns of this paper into proper context. We identified two major signal processing problems of the display end. The first is the computation of the field arising from a 3D scene, starting from its abstract representation. The second is the determination of the drive signals for the display, starting from the field computed in the first. Successful solution of both problems will enable us to drive a display in order to recreate the optical field that would have been generated from the original scene, given its abstract representation.

Different options in computer generated holography were surveyed. Various modern signal processing tools relevant to optical and holographic problems were discussed. While the coverage and scope of the present paper is not comprehensive, one important conclusion that clearly emerges is that the accomplishment of highperformance 3DTV or similar systems will involve not only high-performance display devices, but also the application of sophisticated signal processing approaches and tools and the development of efficient algorithms based on them. While the purely mathematical foundations of these tools are well established, there seems to be considerable work waiting to be done to exploit their full potential in problems in optics and holography.

Acknowledgement: Authors thank Thomas Kries for his comments.

\section{REFERENCES}

1. T. Sikora L. Onural and A. Smolic. An overview of a new european consortium: Intregrated threedimensional television - capture, transmission and display (3DTV). In European Workshop on the Integration of Knowledge, Semantics and Digital Media Technology (EWIMT'04), pages 375-382, Nov 2004.

2. K. Maen. Electro-holographic display using 15 mega pixels lcd. In SPIE, volume 2652, pages 15-23, 1996.

3. P. Aswendt T. Kreis and R. Höfling. Hologram reconstruction using a digital micromirror device. Opt. Eng., 40:926-933, 2001.

4. W. J. Dallas. The Computer in Optical Research, Editor: B. R. Frieden, volume 41 of Springer Series Topics in Applied Physics, pages 291-366. Springer-Verlag, 1980.

5. L.P.Yaroslavsky. Digital Holography and Digital Image Processing: Principles, Methods, Algorithms. Kluwer Academic Publishers, 2003. 
6. D. Leseberg and C. Frère. Computer-generated holograms of 3-d objects composed of tilted planar segments. Appl. Opt., 27:3020-3024, 1988.

7. C. Frère and D. Leseberg. Large objects reconstructed from computer-generated holograms. Appl. Opt., 28:2422-2425, 1989.

8. J. Rosen. Computer-generated holograms of images reconstructed on curved surfaces. Appl. Opt., 38:61366140, 1999.

9. Y. Takaki and H. Ohzu. Hybrid holographic microscopy: Visualization of three-dimensional object information by use of viewing angles. Appl. Opt., 39:5302-5308, 2000.

10. D. Abookasis Y. Li and J. Rosen. Computer-generated holograms of three-dimensional realistic objects recorded without wave interference. Appl. Opt., 40:2864-2870, 2001.

11. Y. Sando, M. Itoh, and T. Yatagai. Holographic three-dimensional display synthesized from threedimensional Fourier spectra of real existing objects. Opt. Lett., 28(24):2518-2520, 2003.

12. Y. Sando, M. Itoh, and T. Yatagai. Color computer-generated holograms from projection images. Opt. Express, 12(11):2487-2493, 2004.

13. D. Abookasis and J. Rosen. Computer-generated holograms of three-dimensional objects synthesized from their multiple angular viewpoints. J. Opt. Soc. Am. A, 20:1537-1545, 2003.

14. Y. Sando, M. Itoh, and T. Yatagai. Full-color computer-generated holograms using 3-d Fourier spectra. Opt. Express, 12(25):6246-6251, 2004.

15. Y. Sando, M. Itoh, and T. Yatagai. Fast calculation method for cylindrical computer-generated holograms. Opt. Express, 13(5):1418-1423, 2005.

16. D. Leseberg. Computer-generated three-dimensional image holograms. Appl. Opt., 31(2):223-229, 1992.

17. T. Ito, H. Eldeib, K. Yoshida, S. Takahashi, T. Yabe, and T.Kunugi. Special purpose computer for holography horn-2. Comput. Phys. Commun., 93:13-20, 1996.

18. Ritter, J. Boettger, O. Deussen, M. Koenig, and T. Strothotte. Hardware-based rendering of full-parallax synthetic holograms. Appl. Opt., 38(8):1364-1369, 1999.

19. C. Petz and M. Magnor. Fast hologram synthesis for 3d geometry models using graphics hardware. Proc. SPIE, 5005:266-275, 2003.

20. M. Lucente. Interactive computation of holograms using a look-up table. J. Electr. Imag., 2(1):28-34, 1993.

21. W. Plesniak. Incremental update of computer generated holograms. Opt.Eng., 42(6):1560-1572, 2003.

22. K. Matsushima and M.Takai. Recurrence formulas for fast creation of synthetic three-dimensional holograms. Appl. Opt., 39(35):6587-6594, 2000.

23. M. Lucente. Diffraction-Specific Fringe Computation for Electro-Holography. PhD thesis, Department of Electrical Engineering and Computer Science, Massachusetts Institute of Technology, September 1994.

24. B. Munjuluri M.L. Huebschman and H.R. Garner. Dynamic holographic 3-d image projection. Opt. Express, 11(5):437-445, 2003.

25. M. L. Huebschman B. Munjuluri and H. R. Garner. Rapid hologram updates for real-time volumetric information displays. Appl. Optics, 44(24):5076-5085, 2005.

26. H. Kim K. Choi and B. Lee. Full-color autostereoscopic 3d display system using color-dispersioncompensated synthetic phase holograms. Opt. Express, 12(21):5229-5236, 2004.

27. H. Godo T. Ito, T. Shimobaba and M. Horiuchi. Holographic reconstruction with a $10 \mu m$ pixel-pitch reflective liquid-crystal display by use of a light-emitting diode reference light. Opt. Lett., 27:1406-1408, 2002.

28. T. Ito and K. Okano. Color electroholography by three colored reference lights simultaneously incident upon one hologram panel. Opt. Express, 12(18):4320-4325, 2004.

29. J.-Y. Son H. Choi, S.-K. Kim and J. Wu. Pulse-laser electroholography by use of interference fringe patterns captured by a ccd. Appl. Optics, 43(30):5600-5607, 2004.

30. H. M. Ozaktas M. Ertosun, H. Atli and B. Barshan. Complex signal recovery from multiple fractional Fourier-transform intensities. Appl. Opt., 44(23):4902-4908, 2005.

31. H. M. Ozaktas M. Ertosun, H. Atli and B. Barshan. Complex signal recovery from two fractional Fourier transform intensities: order and noise dependence. Optics Commun., 244:61-70, 2005.

32. F. Gori. Fresnel transform and sampling theorem. Opt Commun, 39:293-297, 1981. 
33. L. Onural. Sampling of the diffraction field. Appl Opt, 39:5929-5935, 2000.

34. H. M. Ozaktas, Z. Zalevsky, and M. A. Kutay. The Fractional Fourier Transform with Applications in Optics and Signal Processing. Wiley, New York, 2001.

35. G. B. Esmer and L. Onural. Simulation of scalar optical diffraction between arbitrarily oriented planes. In First International Symposium on Control, Communications and Signal Processing, 2004.

36. J. Benedetto and P. Ferreira. Introduction. In J. Benedetto and P. Ferreira, editors, Modern Sampling Theory, pages 1-28. Birkhauser, Boston, 2000.

37. A. Aldroubi and K. Grochenig. Nonuniform sampling and reconstruction in shift-invariant spaces. SIAM Review, 43(4):585-620, 2001.

38. M. Liebling, T. Blu, and M. Unser. Fresnelets: New multiresolution wavelet bases for digital holography. IEEE Transactions on Image Processing, 12:29-43, 2003.

39. I. Daubechies. Time - frequency localization operators : a geometric phase space approach. IEEE Transactions on Information Theory, 34:605-612, 1988.

40. S. Mallat. A Wavelet Tour Of Signal Processing. Academic Press, San Diego, California, 2 edition, 1999.

41. J. J. Benedetto. Ten books on wavelets. SIAM Review, 42(1):127-138, 2000.

42. C. Buraga-Lefebvre, S. Coetmellec, D. Lebrun, and C. Ozkul. Application of wavelet transform to hologram analysis: three-dimensional location of particles. Optics and Lasers in Engineering, 33:409-421, June 2000.

43. D. Lebrun, S. Belaid, and C. Ozkul. Hologram reconstruction by use of optical wavelet transform. Applied Optics, 38(17):3730-3734, June 1999.

44. M. V. Wickerhauser. Adapted Wavelet Analysis from Theory to Software. A K Peters, Wellesley, Massachusetts, 1994.

45. J. Daugmann. Two-dimensional spectral analysis of cortical receptive field profiles. Vision research, 20:847$856,1980$.

46. L. Wiskott, J. M. Fellos, N. Kruger, and C. von der Malsburg. Face recognition by elastic bunch graph matching. IEEE Transactions on Pattern Analysis and Machine Intelligence, 19:775-779, 1997.

47. S. Mann and S. Haykin. Time-frequency perspectives : the chirplet transform. In Proceedings of IEEE Acoustics, Speech, and Signal Processing Conf., pages 417-420, 1992.

48. S. Mann and S. Haykin. The chirplet transform : Physical considerations. IEEE Transactions on Signal Processing, 43:2745-2761, 1995.

49. S. Qian, D. Chen, and Q. Yin. Adaptive chirplet based signal approximation. In Proceedings of IEEE Acoustics, Speech, and Signal Processing Conf., pages 1781-1784, New York, New York, 1998. IEEE.

50. Q. Yin, S. Qian, and A. Feng. A fast refinement for adaptive Gaussian chirplet distribution. IEEE Transactions on Signal Processing, 50:1298-1306, 2002.

51. R. Gribonval. Fast matching pursuit with a multiscale dictionary of Gaussian chirps. IEEE Transactions on signal Processing, 49(5):994-1001, May 2001.

52. S. Qian and D. Chen. Signal representation using adaptive normalized Gaussian functions. IEEE Transactions on Signal Processing, 36:1-11, 1994.

53. L. Angrisani and M. D'Arco. A measurement method based on a modified version of the chirplet transform for instantaneous frequency estimation. IEEE Transactions on Instrumentation and Measurement, 51:704711, August 2002.

54. M. Liebling and M. Unser. Autofocus for digital Fresnel holograms by use of a fresnelet - spar sity criterion. Journal of the Optical Society of America A, 21:2424-2430, 2004. 